Communications in Physics, Vol. 24, No.3S1 (2014), pp. 29-32

DOI:10.15625/0868-3166/24/3S1/5072

\title{
STRUCTURE AND ELECTRICAL PROPERTIES OF THE THIN GOLD LEAVES FABRICATED BY VIETNAM TRADITIONAL LAMINATING TECHNOLOGY
}

\author{
NGUYEN KHAC THUAN AND HOANG NAM NHAT \\ University of Engineering and Technology, Vietnam National University, Hanoi \\ E-mail: namnhat@gmail.com
}

Received 04 June 2014

Accepted for publication 24 August 2014

\begin{abstract}
The structure and electrical properties of the thin gold leaves fabricated by Vietnam traditional laminating technology are introduced. The gold leaves are usually created with the average size of $3 x 3 \mathrm{~cm}$, and 200nm thickness, they can easily be broken when handling carelessly. By the measurements of X-ray Diffraction (XRD), and Electron Scanning Microscopy (SEM), the structure and surface morphology were investigated. We have also measured the absorption spectra and determined the resistivity of the samples and found that the gold films possessed the Ca impurities stuck on the surface of the leaves. The conductivity of the films is relatively higher and the absorption maximum is red-shifted in comparison with that of the bulk.
\end{abstract}

Keywords: ?????????????

\section{INTRODUCTION}

The jewellery that utilizes gold and silver as the main raw materials appeared for a quite long time in Vietnam. The products made up of gold and silver are often very expensive, but those products inlaid with gold leaves are often of much cheaper prices while preserving the basic characteristics of gold which include its color, conductivity and inertness. In the North of Vietnam, the gold laminating technology preserves only in the Kieu Ki village, in the suburb area of Hanoi. The manufacturing technology includes a number of production stages, conducted under a strict order: ink-made, mixed Dzo paper, surf ink on the paper and smash gold into the paper, the finishment etc... Bulk gold is a metal trasmitting a color of yellow, but as known, its shows the different colors in the nanostructures depending on the size of the nanoparticles. It is one of the most malleable and ductile metals. Its electrical and heat conductivity is very high among the metals and is only less than that of the silver and copper. The gold resistivity at room temperature is about $2.27 \times 10^{-8} \Omega$.m [1]. While the bulk gold consists of the particles of $\mu \mathrm{m}$ size, showing the yellow surface color, the gold colloids usually exhibit a much smaller average size of the particles $(5-50 \mathrm{~nm})$, thus, transmitting a color varying from light red $(700 \mathrm{~nm})$ to dark blue $(400 \mathrm{~nm})$ according to the existance of the palsmon resonances at thr surfaces of the nanoparticles $[2,6,7]$. As known, the photoluminescence of a solid gold has a peak around 540nm, this peak shifts to the 
higher frequency in the low temperature [3]. In this paper, we discuss the electrical and optical properties of the thin gold leaves manufactured by the traditional Vietnam hand-made technology, and received from in the Kieu Ki village. The thin gold leaves has an average surface area of $3 \times 3 \mathrm{~cm}$ (Fig. 1), an average mass of $0.00263 \mathrm{~g}$. The measured density was $18.88 \mathrm{~g} / \mathrm{cm}^{3}$, same as reported in Ref. [1], so the average thickness of the leaves was determined to be $200 \mathrm{~nm}$.

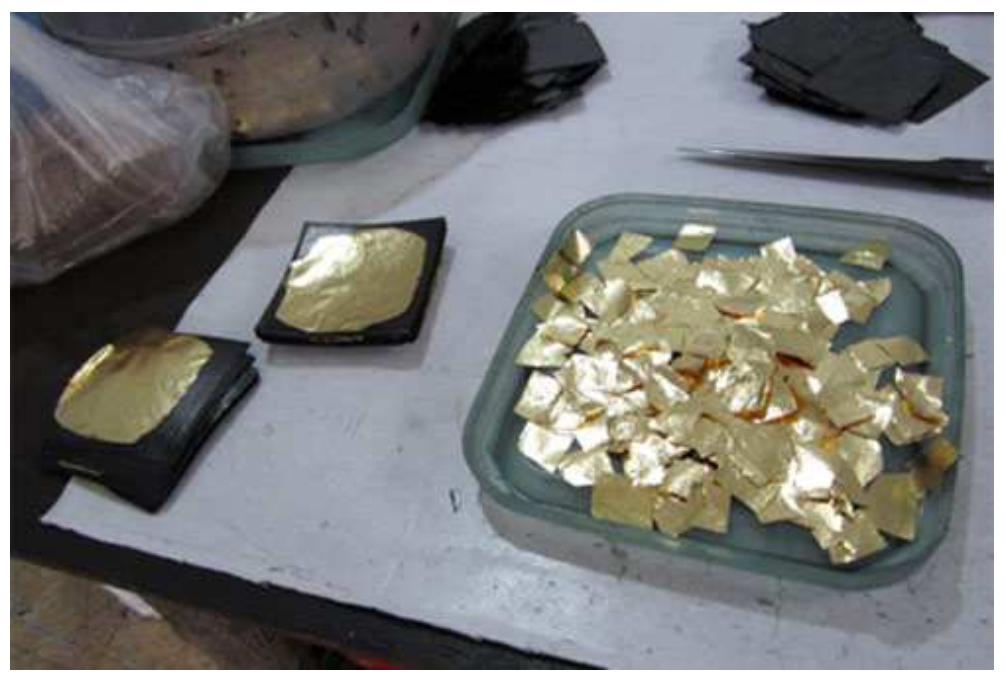

Fig. 1. The thin gold leaves fabricated by the traditional laminating technology.

\section{RESULTS AND DISCUSSION}

Fig. 2 presents the XRD pattern of the gold leaves. The patterns show the maximum diffraction peaks at the specific diffraction angles which should correspond to that of the bulk gold and $\mathrm{Ca}$ (in the $\mathrm{CaCO}_{3}$ structure). The sample appeared to have an orthorhombic structure with the lattice parameters $a=11.58, b=7.746$ and $c=4.883 \AA$. This structure differs from that of the cubic pure bulk gold [4]. However, by the elimination of the $\mathrm{CaCO}_{3}$ phase, and by transformation of the orthorhombic structure to the deformed quasi-cubic lattice, we arrived at the value of $a=4.06 \AA, \beta=89.02^{\circ}$. This result is comparable to that of the bulk [4,5] and the deformation may be assigned to the dimensional effect of the films where the preferred orientation axis appears in the planar structure of the leaves. The Ca impurities occurred only at the surface, and was not substituted for $\mathrm{Au}$ in the lattice.

The surface morphology of the gold leaves were measured by the Scanning Probe Microscopy (CMS, HUS) and the result is showed in the right inset of Fig. 2. we found that the surface of the leaves is fairly uniform, however, there are many spots especially on the edge which may be due to the clamp on where the very thin gold leaves stuck together and could not be separated. The measurement of thickness is showed in the left inset of Fig. 2. There were small grooves on surface and the average thickness was about $240 \mathrm{~nm}$. Following the theoretical calculation, rely on the weight and area of the leaves, its thickness should be $200 \mathrm{~nm}$, that is smaller than the measured result. 


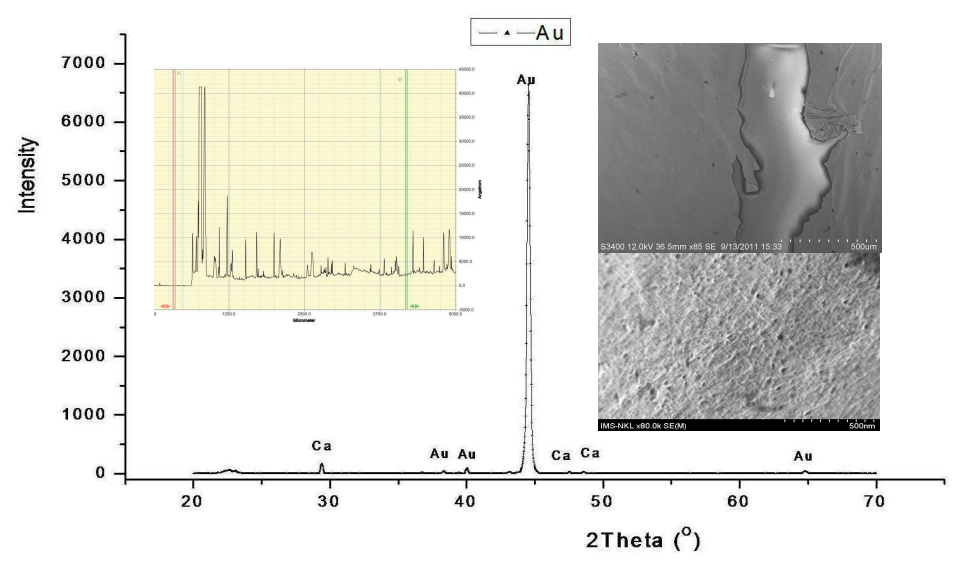

Fig. 2. The XRD patterns of the gold leaves: the inset (left) shows the measurement of thickness and the inset (right) shows the SEM photographs of the surface.

For the optical properties of the leaves we measured the absorption spectra in the range of the wavelength from 200 to $800 \mathrm{~nm}$ (Fig. 3). There are 2 absorption maxima as seen, where one falls in the ultraviolet region, the other appears in the visible region. The maximum has a wavelength of $470 \mathrm{~nm}$, that is in the green range, differs from that of the pure bulk gold [9] and the gold nanoparticles $[6,8]$.
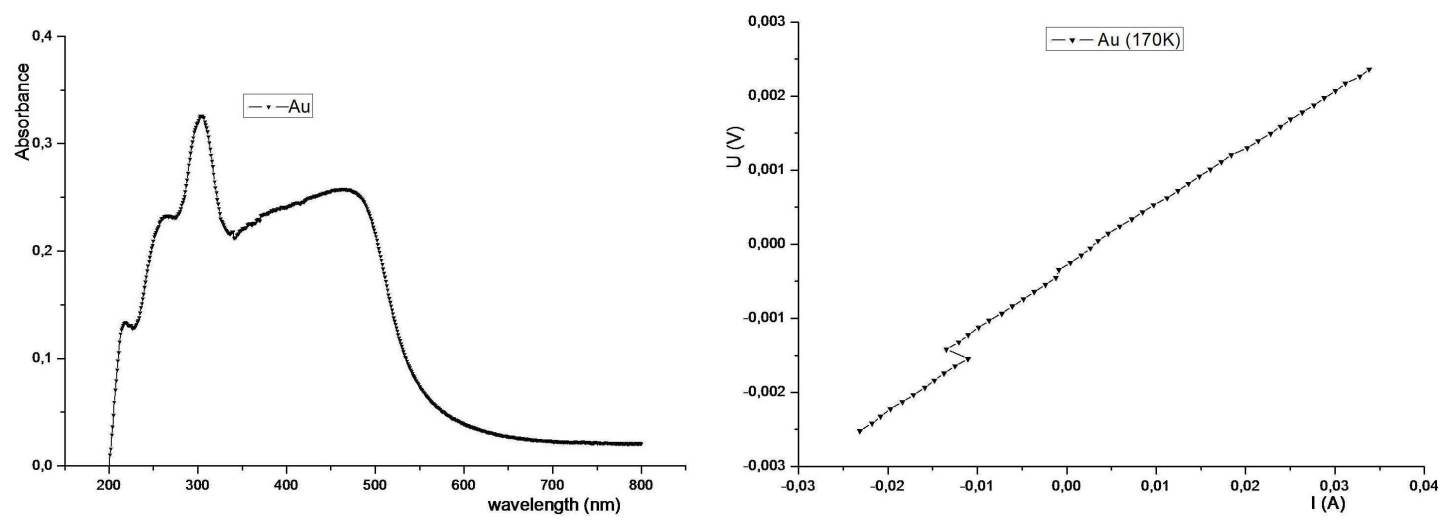

Fig. 3. Absorption spectra and U-I curve of the leaves.

By measuring of the resistance of the leaves at the temperature of $170 \mathrm{~K}$, we found that the U-I curve has a broken track at a voltage of $-1.55 \mathrm{mV}$, the measured resistance at $170 \mathrm{~K}$ was $0.0859 \Omega$, and the resistivity was approximately $6 \times 10^{-9} \Omega \mathrm{m}$, this result is smaller than that of the bulk gold $\left(1.24 \times 10^{-8} \Omega \mathrm{m}\right)$.

\section{CONCLUSION}

The thin gold leaves possess $240 \mathrm{~nm}$ average thickness, with a strong absorption peaks in violet and green regions, thus reflecting a bright yellow color. The leaves have a smaller resistivity 
than that of the solid gold. Currently, the gold leaves laminated by traditional craft technology are still used widely on religious products and fine-arts. The traditional technology has created a very unique product in the sense of optical and electrical properties.

\section{ACKNOWLEDGMENT}

The authors would like to thank the supports from Vietnam National University project code \# QG.12.47.

\section{REFERENCES}

[1] R. A. Matula, J. Phys. Chem. Ref. Data. 8 (4) (1979) 1206-1235.

[2] D. E. Aspnes, E. Kinsbron, and D. D. Bacon, Physical Review B. 21(8) (1980) 135-149.

[3] A. Mooradian, Physical Review Letter. 22(5) (1969) 185-187.

[4] Jason G. Parsons, Jose R. Peralta, Nanomaterials for the Life Sciences. 1 (2009) 461-491.

[5] T. Barth, and G. Lunde, Zeitschrift für Physikalische Chemie 121 (1926) 78-102.

[6] N. A. Bang, P. T. Thom, H. N. Nhat, Gold bull. 46(2) (2013) 91-96.

[7] J. Zhao, , A. O. Pinchuk, J. M. McMahon, S. Li, L. K. Ausman, A. L. Atkinson, and G. C. Schatz. Acc. Chem. Rev. 41 (2008) 1710.

[8] S. K. Ghosh and T. Pal, Chem. Rev. 107 (2007) 4797-4862.

[9] R. Ballerstadt, J. S. Schultz, Anal. Chem. Rev. 72 (2000) 4185. 